\title{
Porous Carbon Microspheres, an Excellent Support to Prepare Surface Molecularly Imprinted Polymers for Selective Removal of Dibenzothiophene in Fuel Oil
}

\author{
Lei Qin ${ }^{1,2}$, Xiaorui Jia ${ }^{1,2}$, Yongzhen Yang ${ }^{1,3^{*}}$ and Xuguang Liu ${ }^{1,2 *}$ \\ ${ }^{1}$ Key Laboratory of Interface Science and Engineering in Advanced Materials \\ (Taiyuan University of Technology), Ministry of Education, Taiyuan 030024, China, \\ 2 College of Chemistry and Chemical Engineering, Taiyuan University of \\ Technology, Taiyuan 030024, China, \\ 3 Research Center of Advanced Materials Science and Technology, Taiyuan \\ University of Technology, Taiyuan 030024, China. \\ *liuxuguang@tyut.edu.cn; yyztyut@126.com
}


Table S1. Surface Area and Porous Structures of Supports and Corresponding SMIPs

\begin{tabular}{cccc}
\hline Sample & $\begin{array}{c}\text { Surface area } \\
\left(\mathrm{m}^{2} \mathrm{~g}^{-1}\right)^{[\mathrm{a}]}\end{array}$ & $\begin{array}{c}\text { Pore volume } \\
\left(\mathrm{cm}^{3} \mathrm{~g}^{-1}\right)^{[\mathrm{b}]}\end{array}$ & $\begin{array}{c}\text { Pore size } \\
(\mathrm{nm})^{[\mathrm{c}]}\end{array}$ \\
\hline PCMSs & 607.5 & 0.21 & 1.25 \\
SMIP/PCMSs & 725.3 & 0.56 & 2.56 \\
NIP/PCMSs & 649.8 & 0.25 & 2.35 \\
NCMSs & 9.0 & -- & -- \\
SMIP/NCMSs & 19.6 & 0.05 & 2.77 \\
NIP/NCMSs & 17.4 & 0.04 & 2.76 \\
AC & 1141.5 & 0.23 & 2.10 \\
SMIP/AC & 2045.5 & 1.90 & 3.17 \\
NIP/AC & 1874.7 & 1.35 & 3.17 \\
\hline
\end{tabular}

[a] Calculated by the multipoint BET model from adsorption data. [b] Calculated by the V-t plot method. [c] Calculated by the BJH model from the adsorption branches of the isotherms. 
Table S2. Kinetics Constant for Adsorption of DBT Dissolved in n-Hexane Solution onto SMIP/PCMSs

\begin{tabular}{|c|c|c|c|c|c|c|c|c|}
\hline & \multirow[b]{2}{*}{$\mathrm{T}(\mathrm{K})$} & \multirow{2}{*}{$\begin{array}{c}Q_{e, \exp } \\
\left(\mathrm{mg} \mathrm{g}^{-1}\right)\end{array}$} & \multicolumn{3}{|c|}{ Pseudo-first-order } & \multicolumn{3}{|c|}{ Pseudo-second-order } \\
\hline & & & $\begin{array}{c}Q_{e, c a l} \\
\left(\mathrm{mg} \mathrm{g}^{-1}\right)\end{array}$ & $\begin{array}{c}k_{1} \\
\left(\min ^{-1}\right)\end{array}$ & $\mathrm{R}^{2}$ & $\begin{array}{c}Q_{e, c a l} \\
\left(\mathrm{mg} \mathrm{g}^{-1}\right)\end{array}$ & $\begin{array}{c}k_{2} \\
\left(\mathrm{~g} \mathrm{mg}^{-1} \min ^{-1}\right)\end{array}$ & $\mathrm{R}^{2}$ \\
\hline NIP & 298 & 65.08 & 63.76 & 0.07858 & 0.9919 & 79.05 & 0.00087 & 0.9638 \\
\hline \multirow[t]{3}{*}{ SMIP } & 288 & 110.32 & 109.66 & 0.08829 & 0.9989 & 125.94 & 0.00079 & 0.9820 \\
\hline & 298 & 118.03 & 114.96 & 0.09763 & 0.9968 & 131.92 & 0.00083 & 0.9793 \\
\hline & 308 & 121.98 & 120.42 & 0.11044 & 0.9956 & 140.85 & 0.00077 & 0.9405 \\
\hline
\end{tabular}


Table S3. Parameters of Isothermal Adsorption Equations for Experimental Adsorption Data

\begin{tabular}{|c|c|c|c|c|c|c|}
\hline \multirow[b]{2}{*}{$T(\mathrm{~K})$} & \multicolumn{3}{|c|}{ Langmuir } & \multicolumn{3}{|c|}{ Freundlich } \\
\hline & $\begin{array}{c}Q_{m} \\
\left(\mathrm{mg} \mathrm{g}^{-1}\right)\end{array}$ & $\begin{array}{c}k_{L} \\
\left(\mathrm{~L} \mathrm{mmol}^{-}\right.\end{array}$ & $\mathrm{R}^{2}$ & $\mathrm{n}$ & $k_{F}$ & $\mathrm{R}^{2}$ \\
\hline 288 & 150.30 & 0.575 & 0.9872 & 1.335 & 3.612 & 0.9774 \\
\hline 298 & 154.92 & 1.237 & 0.9885 & 1.214 & 5.750 & 0.9775 \\
\hline \multirow[t]{2}{*}{308} & 169.28 & 2.158 & 0.9818 & 2.488 & 3.343 & 0.9275 \\
\hline & \multicolumn{3}{|c|}{ Dubinin-Radushkevich } & \multicolumn{3}{|c|}{ Scatchard } \\
\hline$T(\mathrm{~K})$ & $\begin{array}{c}Q_{m} \\
\left(\mathrm{mg} \mathrm{g}^{-1}\right)\end{array}$ & $\begin{array}{c}\beta \\
\left(\mathrm{mol}^{2} \mathrm{~kJ}^{-2}\right.\end{array}$ & $\mathrm{R}^{2}$ & $\begin{array}{c}k_{S} \\
\left(\mathrm{mg} \mathrm{g}^{-1}\right)\end{array}$ & $\begin{array}{c}b \\
\left(\mathrm{mmol} \mathrm{L}^{-1}\right)\end{array}$ & $\mathrm{R}^{2}$ \\
\hline 288 & 218.61 & 0.0362 & 0.9988 & 499.50 & 1.433 & 0.4592 \\
\hline 298 & 254.83 & 0.0290 & 0.9887 & 580.68 & 1.781 & 0.4653 \\
\hline 308 & 223.61 & 0.0122 & 0.9711 & 195.14 & 26.026 & 0.9132 \\
\hline
\end{tabular}


Table S4. Selective Adsorptions of SMIP/PCMSs Towards DBT

Beyond BT, Biphenyl and Fluorene

\begin{tabular}{|c|c|c|c|c|c|c|c|}
\hline \multirow{2}{*}{$\begin{array}{l}\text { Mixed } \\
\text { solution }\end{array}$} & \multicolumn{3}{|c|}{ SMIP/PCMSs } & \multicolumn{3}{|c|}{ NIP/PCMSs } & \multirow{2}{*}{$K^{\prime}$} \\
\hline & $Q_{e}\left(\mathrm{mg} \mathrm{g}^{-1}\right)$ & $K_{d}\left(\mathrm{~L} \mathrm{~mol}^{-1}\right)$ & $K$ & $Q_{e}\left(\mathrm{mg} \mathrm{g}^{-1}\right.$ & $V_{d}\left(\mathrm{~L} \mathrm{~mol}^{-1}\right)$ & $K$ & \\
\hline DBT & 98.76 & 2.0301 & & 47.77 & 0.4794 & & \\
\hline BT & 32.36 & 0.4315 & 4.7051 & 24.04 & 0.2885 & 1.6617 & 2.83 \\
\hline Biphenyl & 24.78 & 0.2513 & 8.0768 & 13.93 & 0.1273 & 3.7666 & 2.14 \\
\hline Fluorene & 18.53 & 0.1619 & 12.5384 & 10.25 & 0.0835 & 5.7404 & 2.18 \\
\hline
\end{tabular}




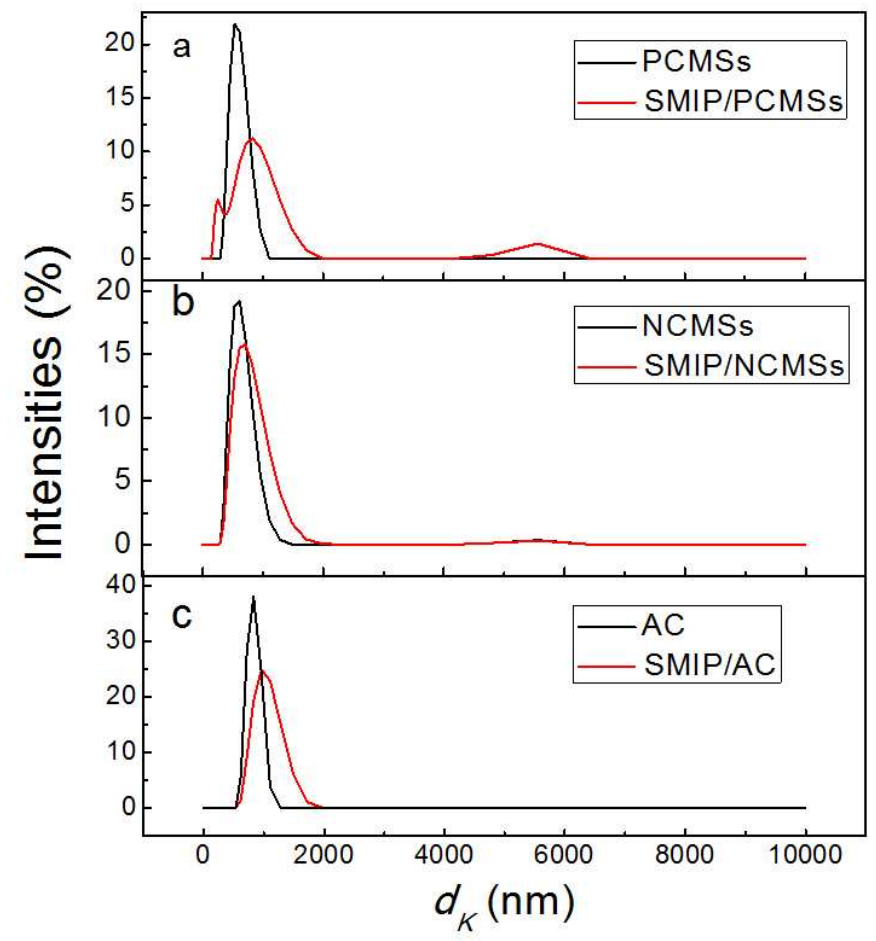

Figure S1. Size distribution obtained by DLS of (a) PCMSs and SMIP/PCMSs, (b) NCMSs and SMIP/NCMSs, (c) AC and SMIP/AC. 


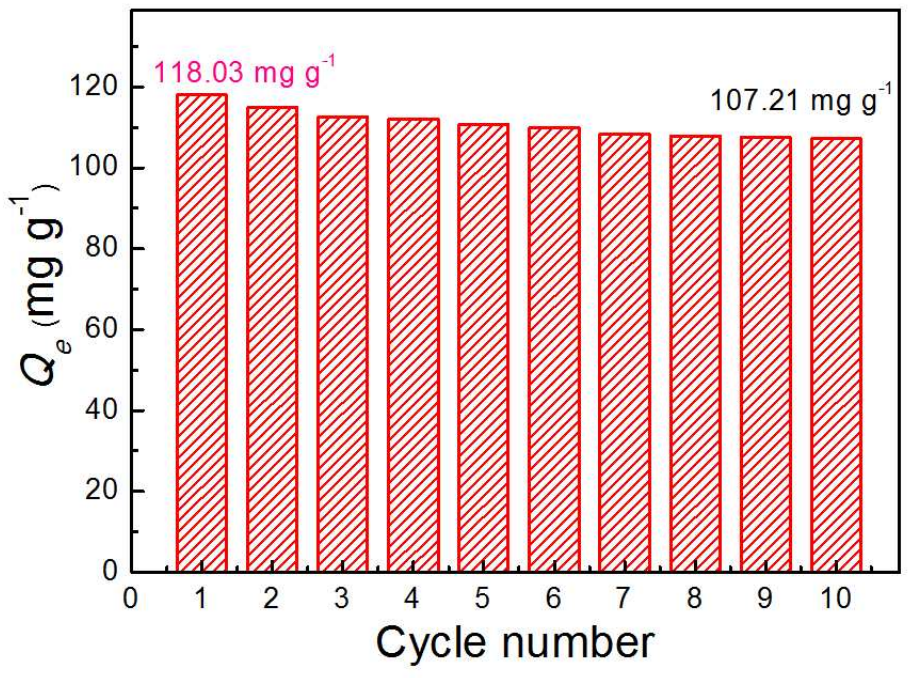

Figure S2. Regeneration of SMIP/PCMSs for DBT adsorption. 